\title{
FORMAS DE APLICAÇÃO DE MICRONUTRIENTES NA CULTURA DA CANA-DE-AÇUCAR
}

VAZQUEZ, Gisele Herbst ${ }^{1}$ SANCHES, Andréa Cristina ${ }^{2}$

Recebido em: 2009.11 .03

Aprovado em: 2010.02.17

ISSUE DOI: $10.3738 / 1982.2278-315$

RESUMO: A adubação é um dos fatores que determinam a produtividade. As plantas necessitam tanto de macro quanto de micronutrientes, visto que esses elementos desempenham funções vitais em seu metabolismo. Porém, a utilização de micronutrientes em cana-de-açúcar ainda apresenta muitas controvérsias. O objetivo deste experimento foi avaliar a eficiência da utilização de micronutrientes na cultura da cana-de-açúcar (cana planta e de ano) fornecido via tolete e foliar. O experimento foi realizado em Campina Verde/MG no período de novembro/2006 a novembro/2007, utilizando-se a variedade RB925345. Os seguintes tratamentos foram avaliados: 1) CanaMicros® pulverizado no tolete + adubação foliar com B, Cu, Mo e Zn (aos 45 dias após o plantio); 2) CanaMicros ${ }^{\circledR}$ via tolete; 3) Micronutrientes via foliar (B, Cu, Mo e Zn) e 4) Testemunha (sem micronutrientes). Os nutrientes aplicados nos toletes foram pulverizados com o inseticida Fipronil no sulco de plantio. Não houve interferência dos tratamentos no número e no diâmetro de colmos, na altura de plantas e nas análises tecnológicas. As produções de cana (TCH) e de pol por hectare (TPH) apresentaram diferenças significativas, o tratamento 1 superou estatisticamente os tratamentos 3 e 4, mas não diferiu do 2. Concluiu-se que o uso de micronutrientes proporciona acréscimos na produtividade da cana-de-açúcar. Melhores resultados são obtidos quando se utilizam aplicações via tolete mais a foliar. A aplicação de micronutrientes via tolete é uma prática eficiente, pois possibilita o uso conjunto com inseticidas, o que diminui os custos e proporciona uma distribuição uniforme dos nutrientes.

Palavras-chave: Micronutrientes. Cana-de-açúcar.

\section{METHODS OF APPLICATION OF MICRONUTRIENTS IN THE CULTURE OF CANE SUGARCANE}

SUMMARY: One of the factors that determines productivity is the fertilization. Plants need macro and micro nutrients because these elements have vital function in their metabolism. However there is still a big controversy over the use of micro nutrients to sugar cane. The goal of this paper was to evaluate the efficiency of the use micro nutrients in sugar cane crop (plant cane and year) given by tolete and foliar. The experiment was made in Campina Verde / MG between November/2006 and November/2007 using the RB925345. The following treatments were analised: 1) CanaMicros ${ }^{\circledR}$ sprayed in seedpieces + foliar fertilization with $\mathrm{B}, \mathrm{Cu}, \mathrm{Mo}$ and $\mathrm{Zn}$ (plants with 45 days); 2) CanaMicros ${ }^{\circledR}$ sprayed throught seedpieces; 3) Micro nutrients throught foliar (the same as treatment 1 after 45 days) 4) witness (without micro nutrients). They sprayed all treatments on Fipronil pesticide. The treament didn't interfere with numbers and diametes of the stalk nor did with the plant hight or technological analysis. The sugar cane production and of pol per hectare show meaning differences. The treatments 1 was statiscally beyond the treaments 3 and 4, but was no diferent of the treatment 2 . In conclusion the use of micro nutrients give an increasing of the sugar cane productivity. We have better results using them throught tolete plus foliar. The application of micro nutrients throught tolete is an efficient practice because we can use it with pesticides, which decreases the costs and gives an equitable distribution of nutrients.

Keywords: Sugarcane. Micronutrients.

\footnotetext{
${ }^{1}$ Doutora em Agronomia (FCAV/UNESP). Docente do Departamento de Fitotecnia, Tecnologia de Alimentos e Sócio Economia, Faculdade de Engenharia, UNESP, CEP 15385-000, Ilha Solteira, SP e do Departamento de Produção Vegetal, Faculdade de Agronomia, Universidade Camilo Castelo Branco, CEP 15600-000, Fernandópolis, SP, e-mail: gisele-agro@uol.com.br

${ }^{2}$ Coordenadora do curso de Agronomia - Universidade Camilo Castelo Branco.
} 


\section{INTRODUÇÃO}

Com a evolução dos tempos, ficou nítido para a agricultura mundial que a adubação é um dos fatores que determinam a produtividade.

A alta produção de cana-de-açúcar e o crescimento do setor a partir da década de 70, foram alguns dos fatores que contribuíram para a expansão e melhoria da produção no Brasil. Essa expansão ocorreu em áreas tradicionais e não tradicionais de cultivo. Muitas indústrias foram montadas em regiões de solos com baixa fertilidade, nos quais, além da necessidade de calagem, adubação com macronutrientes e rotação de culturas, observa-se baixos teores de micronutrientes (VITTI; OLIVEIRA; QUINTINO apud SEGATO; PINTO; JENDIROBA, 2006).

A utilização de micronutrientes em cana-de-açúcar está relacionada à essencialidade desses elementos para as plantas e suas funções no metabolismo das mesmas, aos sintomas visuais de deficiência observados a campo em plantas com suprimento inadequado destes elementos, segundo a técnica da diagnose foliar, em comparação com teores de plantas sadias e de canaviais com altas produtividades; aos baixos teores no solo, principalmente nos arenosos, com baixo teor de matéria orgânica e sem a utilização de resíduos da própria indústria canavieira ou de outras fontes orgânicas e as novas variedades mais produtivas e mais exigentes em micronutrientes (VITTI et al apud RIPOLI et al. 2006).

A carência de micronutrientes na cana-de-açúcar acarreta reduções de produtividade e, possivelmente, a morte das plantas. A importância dos micronutrientes em programas de adubação foi fundamentada em função das quantidades extraídas dos solos. Essas quantidades podem ser baixas $\left(\mathrm{g} \mathrm{ha}^{-1}\right)$, mas de grande importância para o desenvolvimento da planta, podendo, em condições de baixa disponibilidade no solo, se tornar limitante para o desenvolvimento adequado da cultura. Para regiões de solo de baixa fertilidade ou que são explorados durante muitos anos, a ocorrência de deficiência de micronutrientes pode ser agravada. Portanto, a busca de maior produtividade e uma vida útil maior para o canavial torna a adubação com micronutrientes prática fundamental (CASARIN; VILLA NOVA; FORLI apud MARQUES, 2006).

Em relação aos micronutrientes, a cultura da cana-de-açúcar extrai 149 e 86 g de boro; 234 e 105 g de cobre; 1393 e 5525 g de ferro; 1052 e 1420 g de manganês e 369 e 223 g de zinco para produzir $100 \mathrm{t}$ de colmos industrializáveis e de folhas, respectivamente (ORLANDO FILHO apud CÂMARA; OLIVEIRA, 1993).

A aplicação de micronutrientes na cana-de-açúcar é prática pouco utilizada, mesmonas áreas onde os teores no solo são baixos, havendo um reduzido número de trabalhos 
Muitos dos resultados de pesquisa são contraditórios, isso porque existem alguns motivos que podem interferir na resposta à adubação com micronutrientes (DEMATTÊ, 2004; CASARIN; VILLA NOVA; FORLI apud MARQUES, 2006). Tipo de solo, variedade cultivada, idade da planta (ORLANDO FILHO; ROSSETTO; CASAGRANDE apud FERREIRA et al., 2001), áreas onde o canavial não tenha alcançado o máximo potencial produtivo, havendo macronutrientes em quantidades insuficientes; uso de calcário que contém micronutrientes em sua composição ou de resíduos orgânicos (composto, torta-de-filtro, vinhaça, cinzas de caldeira e outros materiais) são exemplos desses motivos (DEMATTÊ, 2004; CASARIN; VILLA NOVA; FORLI apud MARQUES, 2006).

Portanto é necessário avaliar diversos aspectos fitotécnicos relacionados à produção da cultura da cana-de-açúcar para que seja possível determinar o uso ou não de micronutrientes. A aplicação dos micronutrientes pode ser feita de três formas: via solo, via foliar e via tolete, porém, existem ainda poucos estudos comparativos desses diversos modos de fornecimento.

O objetivo deste experimento foi avaliar a eficiência do uso de micronutrientes na cultura da cana-de-açúcar (cana planta e de ano) fornecido via tolete e via foliar.

\section{MATERIAL E MÉTODOS}

O experimento foi conduzido na Escola Municipal Agrícola Adolfo Alves de Resende, no município de Campina Verde/MG, localizada a 19:32’:08' de latitude Sul, na longitude de 49 :29':11' WGr e a uma altitude de $494 \mathrm{~m}$ em um solo cujas características químicas, na profundidade de 0-0,20 e de 0,20-0,40 m, encontram-se nas Tabelas 1 e 2, respectivamente. A análise de textura apresentou um valor de argila de $17 \%$.

Tabela 1 - Características químicas do solo na camada 0-0,20 m antes da instalação do experimento na área. Campina Verde, MG (2006/07).

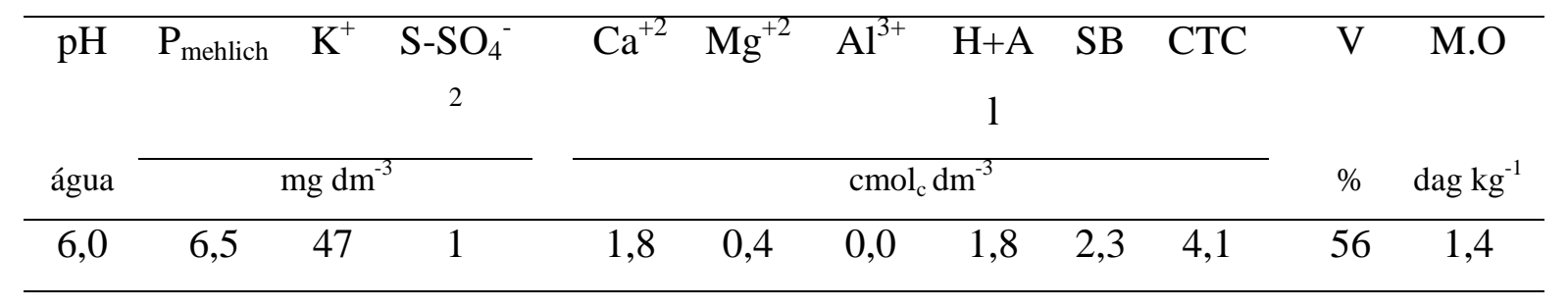

\begin{tabular}{|c|c|c|c|c|c|c|c|c|}
\hline \multicolumn{4}{|c|}{ Relação entre as bases } & B & $\mathrm{Cu}$ & $\mathrm{Fe}$ & $\mathrm{Mn}$ & $\mathrm{Zn}$ \\
\hline $\mathrm{Ca} / \mathrm{Mg}$ & $\mathrm{Ca} / \mathrm{K}$ & $\mathrm{Mg} / \mathrm{K}$ & $\mathrm{Ca}+\mathrm{Mg} / \mathrm{K}$ & & & $\operatorname{ng~d}$ & & \\
\hline 4,5 & 15,0 & 3,3 & 18,0 & 0,13 & 0,7 & 12 & 1,9 & 0,9 \\
\hline
\end{tabular}

Análise realizada no Laboratório de Fertilidade do Solo da Universidade Federal de Uberlândia/MG. 
Tabela 2 - Características químicas do solo na camada 0,20-0,40 m antes da instalação do experimento na área. Campina Verde, MG (2006/07).

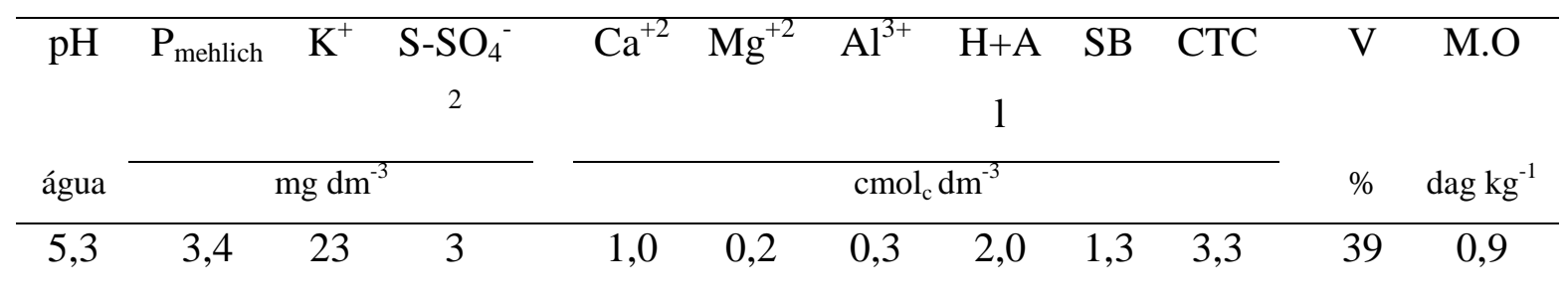

\begin{tabular}{|c|c|c|c|c|c|c|c|c|}
\hline \multicolumn{4}{|c|}{ Relação entre as bases } & B & $\mathrm{Cu}$ & $\mathrm{Fe}$ & $\mathrm{Mn}$ & $\mathrm{Zn}$ \\
\hline $\mathrm{Ca} / \mathrm{Mg}$ & $\mathrm{Ca} / \mathrm{K}$ & $\mathrm{Mg} / \mathrm{K}$ & $\mathrm{Ca}+\mathrm{Mg} / \mathrm{K}$ & \multicolumn{5}{|c|}{$\mathrm{mg} \mathrm{dm}^{-3}$} \\
\hline 5,0 & 16,7 & 3,3 & 20,0 & 0,09 & 0,5 & 8 & 0,3 & 0,1 \\
\hline
\end{tabular}

Análise realizada no Laboratório de Fertilidade do Solo da Universidade Federal de Uberlândia/MG.

Inicialmente a área foi gradeada e após o cálculo da necessidade de calagem na camada 0-0,40 m, de acordo com a fórmula proposta por Vitti et al. (2006a), procedeu-se a distribuição do calcário dolomítico com $85 \%$ de PRNT utilizando-se um equipamento pendular regulado para $2 \mathrm{t} \mathrm{ha}^{-1}$. O calcário foi incorporado com uma grade niveladora em setembro de 2006.

No dia 15/11/2006 a área foi demarcada e procedeu-se a abertura mecanizada dos sulcos de plantio na profundidade de $0,35 \mathrm{~m}$. A adubação foi manual utilizando-se $400 \mathrm{~kg} \mathrm{ha}^{-1}$ da fórmula 4-30-16 e o plantio foi efetuado em sistema de cana de ano com mudas da variedade RB92-5345 com 12 meses de idade procedentes de uma área da Escola Agrícola. As mudas foram distribuídas em sistema "pé com ponta" cruzado, obtendo-se de 13 a 15 gemas por metro. Em seguida, os toletes foram cortados com $40 \mathrm{~cm}$ de comprimento e todas as parcelas pulverizadas com o inseticida Fipronil (Regent 800 WG) na dose de $300 \mathrm{~g}$ do produto comercial por hectare. De acordo com o tratamento estudado, pulverizou-se sobre o tolete, no sulco, o produto Canamicros ${ }^{\circledR}$ Max conjuntamente com o Fipronil. Os sulcos foram cobertos manualmente com uma camada de $5 \mathrm{~cm}$ de solo.

$\mathrm{O}$ experimento foi instalado em delineamento de blocos ao acaso, com quatro tratamentos e cinco repetições. As parcelas possuíam 7 linhas de $10 \mathrm{~m}$ de comprimento no espaçamento de 1,40 m totalizando $98 \mathrm{~m}^{2}$, sendo as cinco linhas centrais, descontando-se 1,0 $\mathrm{m}$ de ambas as extremidades, consideradas como úteis.

Os seguintes tratamentos foram avaliados: 1) CanaMicros® Max via tolete + micronutrientes via foliar (Profol Boro 10®, Profol Cu A/C®, Profol Zn® e Profol Mol 12®) aos 45 dias após o plantio 2) CanaMicros® Max pulverizado no tolete, 3) Adubação foliar aos 45 DAP (Profol Boro 10®, Profol Cu A/C®, Profol Zn® e Profol Mol 12®) e 4) Testemunha (sem micronutrientes). As doses e a composição dos produtos empregados estão apresentadas 
na Tabela 3, e na Tabela 4, encontram-se os elementos minerais e as quantidades fornecidas de acordo com o tratamento estudado.

Tabela 3 - Doses e especificações dos produtos empregados no experimento.

\begin{tabular}{|c|c|c|c|c|c|c|c|}
\hline \multirow{2}{*}{ PRODUTOS } & \multirow{2}{*}{ Dose } & $\mathrm{B}$ & $\mathrm{Cu}$ & Mo & $\mathrm{Zn}$ & $\mathrm{S}$ & Mn \\
\hline & & \multicolumn{6}{|c|}{$\%$} \\
\hline CanaMicros@ Max & $10 \mathrm{~kg} \mathrm{ha}^{-1}$ & 5 & 4,5 & 2 & 11 & 10 & 4,5 \\
\hline Profol Boro 10® & $2,5 \mathrm{~L}$ ha & 10,0 & - & - & - & - & - \\
\hline Profol $\mathrm{Cu} A / C \circledR$ & $0,5 \mathrm{~L} \mathrm{ha}^{-1}$ & - & 6,0 & - & - & - & - \\
\hline Profol Zn® & $0,6 \mathrm{~L} \mathrm{ha}^{-1}$ & - & - & - & $20,0-21,0$ & - & - \\
\hline Profol Mol 12® & $0,4 \mathrm{~L} \mathrm{ha}^{-1}$ & - & - & 12,0 & - & - & - \\
\hline
\end{tabular}

Análises efetuadas pelo Laboratório da empresa Produquímica Ind. e Com Ltda. (2006).

Tabela 4 - Elementos minerais e quantidades de micronutrientes fornecidos de acordo com o tratamento estudado. Campina Verde, MG (2006/07).

\begin{tabular}{|c|c|c|c|c|c|c|c|}
\hline \multirow{2}{*}{\multicolumn{2}{|c|}{ TRATAMENTOS }} & $\mathrm{B}$ & $\mathrm{Cu}$ & Mo & $\mathrm{Zn}$ & $\mathrm{S}$ & $\mathrm{Mn}$ \\
\hline & & \multicolumn{6}{|c|}{ g do elemento por ha } \\
\hline 1- $\quad$ CanaMicros ${ }^{\circledR} \quad$ no tolete & + & 750 & 480 & 248 & 1220 & 1000 & 450 \\
\hline Adubação foliar & & & & & & & \\
\hline 2 - CanaMicros $®$ no tolete & & 500 & 450 & 200 & 1100 & 1000 & 450 \\
\hline 3 - Adubação Foliar & & 250 & 30 & 48 & 120 & - & - \\
\hline 4 - Testemunha & & - & - & - & - & - & - \\
\hline
\end{tabular}

Vinte dias após o plantio aplicou-se o herbicida Diuron (Karmex) na dose de $3 \mathrm{~kg}$ do produto comercial por hectare em área total em pós-emergência inicial. Posteriormente, realizou-se a capina manual em áreas onde houve necessidade.

A adubação de cobertura foi realizada manualmente 45 DAP, utilizando-se $40 \mathrm{~kg} \mathrm{ha}^{-1}$ de nitrogênio na forma de sulfato de amônio e $45 \mathrm{~kg} \mathrm{ha}^{-1}$ de potássio na forma de cloreto de potássio. No mesmo dia, procedeu-se a adubação foliar com os produtos Profol Boro 10® + Profol Zn® + Profol Cu A/C® + Profol Mol 12® nas parcelas do tratamento 3.

Não houve incidência elevada de pragas e doenças na área. Os dados pluviométricos do período do experimento encontram-se na Tabela 5. A colheita foi feita manualmente aos 365 dias após o plantio.

Tabela 5 - Dados pluviométricos em mm. Campina Verde, MG (2006/07).

\begin{tabular}{cccccccccccccc}
\hline Ano & Jan & Fev & Mar & Abr & Mai & Jun & Jul & Ago & Set & Out & Nov & Dez & Total \\
\hline 2006 & 270 & 288 & 170 & 29 & 28 & 0 & 0 & 51 & 71 & 18 & 72 & 105 & 1102 \\
2007 & 401 & 225 & 33 & 67 & 65 & 0 & 48 & 0 & 0 & 17 & 41 & 0 & 897 \\
\hline
\end{tabular}

Dados obtidos na Escola Adolfo Alves de Resende, Campina Verde/MG.

As seguintes determinações foram realizadas: 
- número de colmos por metro: contagem de todos os colmos industrializáveis em 3 linhas de $3 \mathrm{~m}$ dentro da parcela útil aos 30,180 e 365 DAP (colheita);

- altura de colmos: foram medidos 10 colmos industrializáveis dentro da parcela útil entre o ponto de corte e o ponto de quebra do palmito aos 180 e 365 DAP;

- diâmetro de colmos: determinação efetuada no terço inferior de 15 colmos industrializáveis dentro da parcela útil na época da colheita;

- produtividade agrícola expressa em toneladas de colmos por hectare (TCH) - corte basal de todos os colmos da parcela útil e eliminação do palmito, pesagem e posterior extrapolação do resultado obtido para 1 hectare;

- análises tecnológicas - amostras de 10 colmos por parcela no momento da colheita foram separadas e encaminhadas ao Laboratório da Destilaria Alcoeste (Fernandópolis, SP) para a determinação do brix\%caldo, pol\%cana, fibra\%cana, açúcares redutores (AR\%cana) e ATR (açúcar total recuperável) obedecendo a metodologia proposta pelo Consencana/SP (2006);

- tonelada de pol por hectare (TPH) - determinado através da multiplicação dos valores de TCH por pol\%cana e posterior divisão por 100. Esse parâmetro expressa a produtividade de açúcar por área.

Os dados obtidos foram devidamente tabulados e posteriormente submetidos a análises de variância pelo Teste $F$. Nos casos em que se verificaram efeitos significativos $(P<0,05)$, aplicou-se o teste de Tukey para comparação de médias (PIMENTEL-GOMES; GARCIA, 2002).

\section{RESULTADOS E DISCUSSÃO}

De acordo com os resultados das Tabelas 1 e 2, o solo desse experimento apresenta um teor muito baixo de $\mathrm{B}$ e $\mathrm{Mn}$, baixo de $\mathrm{Cu}$, Fe e $\mathrm{Zn}$ na camada 0 - 0,20 m e um teor muito baixo de B, Fe, Mn e Zn e baixo de Cu na camada 0,20 - 0,40 m, segundo Ribeiro, Guimarães e Alvarez (1999).

Na Tabela 6 estão apresentados os resultados de número de colmos por metro aos 30, 180 e 365 DAP e altura de colmos aos 180 e 365 DAP.

Não houve interferência dos tratamentos estudados sobre o número de colmos aos 30 DAP. Aos 180 DAP, o tratamento 1 (tolete + foliar) apresentou um valor estatisticamente superior aos demais, porém, na colheita, essa diferença não se manteve e a área apresentou uma média de 14,4 colmos $\mathrm{m}^{-1}$. Esse valor foi maior que o relatado por Casagrande (1991) de 12 colmos $\mathrm{m}^{-1}$ como sendo o médio para a cultura da cana-de-acúcar e esteve dentro do descrito por Coleti; Walder; Rodrigues (1987) e Graziano (1988) de 12 a 16 colmos em idade 
de corte por metro linear. A época de plantio e a época do corte da cana planta ou das soqueiras, associadas à maturação das variedades, influi no perfilhamento e no número final de colmos industrializáveis (CÂMARA apud CÂMARA; OLIVEIRA, 1993).

Os tratamentos estudados também não interferiram na altura e no diâmetro de colmos nas épocas avaliadas.

Tabela 6 - Resultados médios referentes a número de colmos por metro, altura de colmos e diâmetro de colmos na colheita. Campina Verde/MG, 2006/07.

\begin{tabular}{|c|c|c|c|c|c|c|}
\hline \multirow{3}{*}{ Tratamento } & \multicolumn{3}{|c|}{$\mathrm{N}^{\circ}$ de colmos $\mathrm{m}^{-1}$} & \multicolumn{2}{|c|}{$\begin{array}{l}\text { Altura de }(\mathrm{m}) \\
\text { colmos }\end{array}$} & \multirow{3}{*}{$\begin{array}{c}\text { Diâmetr } \\
\text { o } \\
(\mathrm{mm})\end{array}$} \\
\hline & 30 & 180 & 365 & 180 & 365 & \\
\hline & \multicolumn{3}{|c|}{ DAP } & \multicolumn{2}{|c|}{ DAP } & \\
\hline $\begin{array}{l}\text { 1. CanaMicros@ } \\
\text { tolete + adub. foliar }\end{array}$ & 9,4 & $11,9 \mathrm{~A}$ & 15,9 & 1,62 & 2,04 & 24,34 \\
\hline $\begin{array}{l}\text { 2. CanaMicros® no } \\
\text { tolete }\end{array}$ & 8,0 & $9,4 \mathrm{~B}$ & 14,4 & 1,62 & 1,95 & 23,45 \\
\hline 3. Adubação foliar & 7,0 & $9,0 \mathrm{~B}$ & 12,9 & 1,58 & 2,04 & 23,65 \\
\hline 4. Testemunha & 8,6 & $9,1 \mathrm{~B}$ & 14,4 & 1,56 & 1,95 & 22,74 \\
\hline Média geral & 8,3 & 9,8 & 14,4 & 1,59 & 2,00 & 23,55 \\
\hline $\mathrm{CV}(\%)$ & 22,05 & 10,62 & 12,56 & 5,54 & 8,09 & 5,03 \\
\hline
\end{tabular}

Médias seguidas pela mesma letra na coluna não diferem entre si, pelo teste de Tukey, ao nível de 5\% de probabilidade.

Os resultados referentes a produtividade agrícola e análise tecnológica estão apresentados na Tabela 7.

A produtividade $(\mathrm{TCH})$ apresentou diferenças significativas, o tratamento 1 (tolete + foliar) superou estatisticamente os tratamentos 3 (foliar) e 4 (testemunha), mas não diferiu do 2 (tolete), o que indica a necessidade do uso de micronutrientes na área devido as baixas disponibilidades dos elementos. O uso de micronutrientes de forma conjugada (tolete + foliar) promoveu acréscimo de 28,5\% (18,5 $\left.\mathrm{t} \mathrm{ha}^{-1}\right)$ em relação à testemunha. Embora não haja diferenças estatísticas quanto ao uso via tolete (tratamento 2 ) e via tolete + foliar (tratamento 1), houve um aumento de 23,8\% (16 t ha $\left.{ }^{-1}\right)$ quando se utilizou as duas aplicações. O uso de fontes solúveis de micronutrientes em aplicações conjuntas com defensivos na cobertura do sulco tem sido utilizado pelos produtores de cana, apresentando resultados positivos (ORLANDO FILHO; ROSSETTO; CASAGRANDE apud FERREIRA et al., 2001), embora, na literatura, não existam trabalhos demonstrando a sua eficiência (CASARIN et al., 2006). Porém, segundo os mesmos autores, alguns trabalhos estão sendo desenvolvidos como o realizado pelo ESALQ Júnior / GAPE / COSAN (não publicado) em Tietê/SP que avaliou o efeito da aplicação de micronutrientes via tolete, via foliar, aplicação conjunta e de uma 
testemunha. A aplicação dos micronutrientes via tolete em cana planta foi realizada com o produto CanaMicros ${ }^{\circledR}$, que correspondeu a $770 \mathrm{~g} \mathrm{ha}^{-1} \mathrm{de} \mathrm{Zn}, 350 \mathrm{~g} \mathrm{ha}^{-1} \mathrm{de} \mathrm{B}, 315 \mathrm{~g} \mathrm{ha}^{-1} \mathrm{de}$ $\mathrm{Cu}, 231 \mathrm{~g} \mathrm{ha}^{-1}$ de Mn e $140 \mathrm{~g} \mathrm{ha}^{-1}$ de Mo. A aplicação foliar foi feita aos 45 dias através do fornecimento de $393 \mathrm{~g} \mathrm{ha}^{-1} \mathrm{de} \mathrm{N}, 163 \mathrm{~g} \mathrm{ha}^{-1} \mathrm{de} \mathrm{Zn}, 202 \mathrm{~g} \mathrm{ha}^{-1} \mathrm{de} \mathrm{B}, 83 \mathrm{~g} \mathrm{ha}^{-1} \mathrm{de} \mathrm{Cu}$ e $154 \mathrm{~g} \mathrm{ha}^{-1}$ de Mo. As respostas mostraram ganhos de produtividade, mesmo em condições de canavial de alta produtividade. $\mathrm{O}$ uso de micronutrientes via tolete promoveu acréscimo de $5 \%\left(8,6 \mathrm{t} \mathrm{ha}^{-1}\right)$ e o da aplicação conjunta de 5,5\% (9,5 $\left.\mathrm{t} \mathrm{ha}^{-1}\right)$ em relação à testemunha. Portanto, a aplicação de micronutrientes no momento da "cobrição" dos toletes, juntamente com a aplicação do inseticida, pode se tornar uma prática importante por dois aspectos principais: eficiência de aplicação e redução de custos operacionais.

São escassos os trabalhos com resposta da cana-de-açúcar aos micronutrientes na como o de Alvarez e Wutke (1963), que em solos muito explorados do Estado de São Paulo verificaram os seguintes acréscimos de produção, em toneladas por hectare: B - 21,6; Mo 12,0; Fe - 11,6 e Cu - 8,3. Por sua vez, Malavolta (1990) citado por Mellis, Quaggio e Cantarella apud Dinardo-Miranda, Vasconcelos e Landell (2008) com duas aplicações foliares de $0,175 \mathrm{~kg} \mathrm{ha}^{-1}$ de $\mathrm{B}$, conseguiram aumentos da ordem de $18 \%$ e Azeredo e Bolsanello (1981) de até $27 \%$ na produtividade da cana planta com o uso de $5 \mathrm{~kg} \mathrm{ha}^{-1}$ no sulco ou pulverizações com solução contendo $5 \mathrm{~g} \mathrm{~L}^{-1}$ de $\mathrm{Mn}$.. A resposta à adubação com micronutrientes é mais comum em solos de tabuleiros no Nordeste do país. Aumentos na produtividade de até $40 \mathrm{t} \mathrm{ha}^{-1}$ com o uso de $\mathrm{Cu}$ foram obtidos por Marinho e Albuquerque (1981) para cana planta em várias unidades do Nordeste brasileiro. Cambia et al., (1989) também observaram respostas positivas da cana-de-açúcar em um latossolo, à aplicação de zinco. Orlando Filho, Macedo e Tokeshi (1994) recomendam o uso de $5 \mathrm{~kg} \mathrm{ha}^{-1}$ de Cu e $7 \mathrm{~kg}$ $\mathrm{ha}^{-1}$ de Zn no sulco de plantio para evitar a deficiência desses nutrientes na cultura.

Em termos médios, a produtividade foi baixa $\left(69,8 \mathrm{t} \mathrm{ha}^{-1}\right)$ estando inclusive abaixo da média de $85 \mathrm{t} \mathrm{ha}^{-1}$ da região Centro-Sul (COELHO; LELIS NETO; CUNHA, 2008), o que demonstra que essa variedade não está adaptada a ambientes rústicos, além de não se comportar adequadamente como cana de ano.

Quanto à análise tecnológica, não houve interferência dos tratamentos estudados nos resultados (Tabela 7). De maneira geral, os valores do brix\%caldo, da pol\%cana e do ATR foram baixos $(17,2 \%, 11,3 \%$ e 111,3 respectivamente) e o dos AR\%cana, elevado $(0,722 \%)$. O mínimo ideal para início de colheita é de $13 \%$ para a pol\%cana e inferior a $0,5 \%$ para os AR\%cana (LANDELL; BRESSIANI, 2008). Esses resultados mostram que a colheita deveria ter sido realizada meses antes, principalmente porque a variedade RB925345 é de maturação precoce, sendo recomendada para as safras de outono e inverno (LANDELL; BRESSIANI, 
2008). Segundo os meses autores, a safra de primavera tem como principal característica a oscilação da qualidade e da produtividade ano a ano, devendo ser alocadas em ambientes favoráveis cultivares rústicas, estáveis, sem florescimento e isoporização e de maturação tardia.

Não obstante os atributos tecnológicos não terem apresentados diferenças, os valores de TPH indicaram a mesma tendência da produtividade (Tabela 7).

Tabela 7 - Resultados médios referentes a tonelada de cana por hectare $(\mathrm{TCH})$, análise tecnológica e tonelada de pol por hectare (TPH). Campina Verde/MG, 2006/07.

\begin{tabular}{|c|c|c|c|c|c|c|c|}
\hline \multirow[b]{2}{*}{ Tratamento } & \multirow[b]{2}{*}{$\mathrm{TCH}$} & \multicolumn{5}{|c|}{ Análise tecnológica $^{1}$} & \multirow[b]{2}{*}{ TPH } \\
\hline & & $\begin{array}{c}\text { Brix \% } \\
\text { caldo }\end{array}$ & $\begin{array}{c}\text { Pol \% } \\
\text { cana }\end{array}$ & $\begin{array}{l}\text { Fibra } \\
\text { \%cana }\end{array}$ & $\begin{array}{c}\text { AR } \\
\% \text { cana }\end{array}$ & ATR & \\
\hline $\begin{array}{l}\text { 1. CanaMicros } ₫ \\
\text { tolete }+ \text { adub.foliar } \\
2 \text { CanaMicros } \AA \text { no }\end{array}$ & $83,2 \mathrm{~A}$ & 16,7 & 10,8 & 14,2 & 0,754 & 106,6 & $9,73 \mathrm{~A}$ \\
\hline tolete & $67,2 \mathrm{AB}$ & 17,2 & 11,2 & 14,5 & 0,726 & 109,9 & $8,30 \mathrm{AB}$ \\
\hline 3. Adubação foliar & $64,0 \mathrm{~B}$ & 17,8 & 12,1 & 14,2 & 0,641 & 117,3 & $7,68 \mathrm{~B}$ \\
\hline 4. Testemunha & $64,7 \mathrm{~B}$ & 17,0 & 11,4 & 13,6 & 0,767 & 111,4 & $7,36 \mathrm{~B}$ \\
\hline Média geral & 69,8 & 17,2 & 11,3 & 14,1 & 0,722 & 111,3 & 8,27 \\
\hline $\mathrm{CV}(\%)$ & 14,06 & 5,68 & 7,57 & 5,52 & 14,1 & 6,69 & 10,05 \\
\hline
\end{tabular}

\section{CONCLUSÃO}

O uso de micronutrientes proporciona acréscimos na produtividade da cana-de-açúcar. Melhores resultados são obtidos quando se utilizam aplicações via tolete mais a foliar. A aplicação de micronutrientes via tolete é uma prática eficiente e economicamente viável pois possibilita o uso conjunto com inseticidas, o que diminui os custos e proporciona uma distribuição mais uniforme dos produtos.

\section{REFERÊNCIAS}

ALVAREZ, R.; WUTKE, A.C.P. Adubação da cana-de-açúcar. IX Experimentos preliminares com micronutrientes. Bragantia, v. 22, p. 647-650, 1963.

AZEREDO, D.F.; BOLSANELLO, J. Efeito de micronutrientes na produção e na qualidade da cana-de-açúcar no Rio de Janeiro, Espírito Santo e Minas Gerais (Zona da Mata) - estudo preliminar. Brasil Açucareiro, v.93, p.9-17, 1981.

CÂMARA, G.M.S. Ecofisiologia da cana-de-açúcar. In: CÂMARA, G.M.S. OLIVEIRA, E.A.M. Produção de cana-de-açúcar. Piracicaba. FEALQ. 1993. p. 31-64.

CASAGRANDE, A.A. Tópicos de morfologia e fisiologia da cana-de-açúcar. Jaboticabal: Funep, 1991. 157p. 
CASARIN, V.; VILLA NOVA, V.S.; FORLI, F. Micronutrientes em cana-de-açúcar. In: MARQUES, M.O. et al. (org.) Tópicos em tecnologia sucroalcooleira. Jaboticabal: Gráfica Multipress, 2006. p. 65-76.

COELHO, R.D.; LELIS NETO, J.A.; CUNHA, A.B. Cana irrigada produz mais, mas custo de produção aumenta. In: AGRIANUAL, 2009. InstitutoFNP, 2008, p. 240-242.

COLETI, J. T.; WALDER, L. A M.; RODRIGUES, J. C. S. Estudo de espaçamentos em duas variedades de cana-de-açúcar: SP70-1143 e NA56-79. STAB: açúcar, álcool e subprodutos, Piracicaba, v. 6, n. 2, p. 32-34, 1987.

CONSECANA. Manual de instruções. Piracicaba, Edição Consecana - SP, 2006, 116p.

DEMATTÊ, J.L.I. Recuperação e manutenção da fertilidade dos solos. Visão agrícola, v.1, p. 48-49, 2004.

GRAZIANO, J. R. Espaçamento reduzido de plantio de cana na Usina Palmeiras S/A. STAB: açúcar, álcool e subprodutos, Piracicaba, v. 7, n. 1, p. 28-32, 1988.

LANDELL, M.G.A.; BRESSIANI, J.A. Melhoramento genético, caracterização e manejo varietal. In: DINARDO-MIRANDA, L.L.; VASCONCELOS, A.C.M.; LANDELL, M.G.A. (Editores) Cana-de-açúcar. Campinas: Instituto Agronômico, 2008. p. 101-155.

MARINHO, M.L.; ALBUQUERQUE, G.A.C. Efeito do cobre e do zinco na produção de cana-de-açúcar em solos de tabuleiros de Alagoas. Brasil Açucareiro, v.98, p. 41-50, 1981.

MELLIS, E.V.; QUAGGIO, J.A.; CANTARELLA, H. Micronutrientes. In: DINARDOMIRANDA, L.L.; VASCONCELOS, A.C.M.; LANDELL, M.G.A. (Editores) Cana-deaçúcar. Campinas: Instituto Agronômico, 2008. p. 331-335.

ORLANDO FILHO, J. Calagem e adubação da cana-de-açúcar. In: CÂMARA, G.M.S.; OLIVEIRA, E.A.M. (Editores). Produção de cana-de-açúcar. Piracicaba: FEALQ/USP, 1993. p. 133-146.

ORLANDO FILHO, J.; MACEDO, N.; TOKESHI, H. Seja o doutor do seu canavial. Piracicaba: POTAFOS, 1994. 16p. (Arquivo do Agrônomo, 6).

ORLANDO FILHO, J.; ROSSETTO, R.; CASAGRANDE, A.A. Cana-de-açúcar: In: FERREIRA, M.E. et al. (Editores). Micronutrientes e elementos tóxicos na agricultura. Jaboticabal: CNPq/FAPESP/POTAFOS, 2001. p. 355-369.

PIMENTEL-GOMES, F; GARCIA, C. H. Estatística aplicada a experimentos agronômicos e florestais. Piracicaba: FEALQ, 2002. 309p.

RIBEIRO, A.C.; GUIMARÃES, P.T.G.; ALVAREZ V., V.H. Comissão de fertilidade do solo do Estado de Minas Gerais, recomendações para o uso de corretivos e fertilizantes em Minas Gerais - 5 aproximação, Viçosa, MG, 1999. 359p.

VITTI, G. C.; MAZZA, J.A.; QUINTINO, T.A.; OTTO, R. Nutrição e adubação. In: RIPOLI, T.C.C.; RIPOLI, M.L.C.; CASAGRANDI, D.V.; IDE, B.Y. Plantio de cana-de-açúcar: estado da arte. Piracicaba: T.C.C. Ripoli, 2006. p. 102-144.

VITTI, G. C.; OLIVEIRA, D.B.; QUINTINO, T.A. Micronutrientes na cultura da cana-deaçúcar. In: SEGATO, S.V., PINTO, A.S., JENDIROBA, E. (org.) Atualização em produção de cana-de-açúcar. Piracicaba: Livroceres, 2006. p. 122-138. 